\title{
Nuevo registro del Ocelote (Leopardus pardalis) para el estado de Puebla
}

\author{
Osvaldo Eric Ramírez Bravo ${ }^{1,2}$, Stephanie Schinkel Brault ${ }^{1}$ \\ y Cristina Hernández Santín ${ }^{1}$
}

\section{Abstract}

In this study, we confirmed the presence of the ocelot (Leoparuds pardalis) for the Sierra Norte of the state of Puebla with camera trapping.

Key words: Camera trapping, Hueytamalco, Ocelot, Puebla, Sierra Norte

\section{Resumen}

En este estudio, mediante cámaras confirmamos la presencia del ocelote (Leopardus pardalis) para la Sierra Norte del estado de Puebla.

Palabras clave: Camaras trampa, Hueytamalco, Ocelote, Puebla, Sierra Norte.

El estudio de los carnívoros en Puebla, ha sido escaso e inclusive la presencia de algunas especies no ha sido claramente confirmada. En el caso de los felinos, existe muy poca información a nivel estatal y se desconoce su distribución exacta dentro del estado. En una revisión sobre los carnívoros de Puebla, Ramírez-Pulido et al. (2005) registraron gato montés (Lynx rufus), puma (Puma concolor), tigrillo o margay (Leopardus wiedii) y jaguarundi (Herpailurus yagouaroundi). Adicionalmente, Villareal et al. (2005) registra la existencia de rastros de jaguar (Panthera onca) y de ocelote (L. pardalis) para el campo experimental Las Margaritas, en la zona de bosque tropical perennifolio en Hueytamalco. López-Wilchis y López Jardines (1999) mencionan la existencia de ocelote en el estado.

Dentro de un estudio sobre "El jaguar en Puebla: presencia y relación con el hombre", se seleccionaron varios sitios para fototrampeo. Se utilizaron dos cámaras trampa de la marca Wildview Xtreme 4, en una zona con fragmentos de Selva Alta Perennifolia en el municipio de Hueytamalco (20 $\left.0140^{\prime \prime} \mathrm{N}, 97^{\circ} 18^{\prime} 11^{\prime \prime} \mathrm{W}\right)$. Las cámaras se colocaron en diferentes sitios dentro del mismo parche, a una altura de $30 \mathrm{~cm}-40 \mathrm{~cm}$ por un periodo de un mes, en senderos y sitios donde se observaron rastros de animales o actividad reciente.

Las cámaras reunieron un total de 246 noches trampa, como resultado se obtuvieron dos fotos de ocelote que corresponderían a los primeros registros fotográficos para el

\footnotetext{
${ }^{1}$ Departamento de Ciencias Químico-Biológicas, Universidad de las Américas, Puebla, Santa Catarina Mártir, Sin Número, Cholula, Puebla. Tel (222) 2-29-20-67, Autor corresponsal:ermex02@yahoo.com

${ }^{2}$ Durrell Institute for Conservation Ecology, Marlowe Building, University of Kent, Canterbury, Kent, CT2 7NR, England
} 
estado de Puebla (Fig. 1). Otras especies presentes en la zona fueron armadillos (Dasypus novemcinctus), hocofaisán (Crax rubra), coyote (Canis latrans), mapache (Procyon lotor) y coatie (Nasua narica). Adicionalmente, se encontraron dos especímenes disecados por personas de las comunidades. Uno pertenece a la comunidad de Jopala en el municipio de Jopala $\left(20^{\circ} 0140^{\prime \prime} \mathrm{N}, 97^{\circ} 41^{\prime} 34^{\prime \prime} \mathrm{W}\right)$ y otro a la comunidad de la Unión, en el municipio de Zihuatéutla $\left(20^{\circ} 16^{\prime} 01^{\prime \prime} \mathrm{N}, 97^{\circ} 53^{\prime} 02^{\prime \prime} \mathrm{W}\right)$ ambas en el norte del Estado.

Las fotos de ocelote obtenidas indican la existencia de una población a lo largo de la Sierra Norte del estado de Puebla. Sin embargo, es necesario generar información sobre la distribución y estado de la misma para poder generar medidas adecuadas para su conservación.

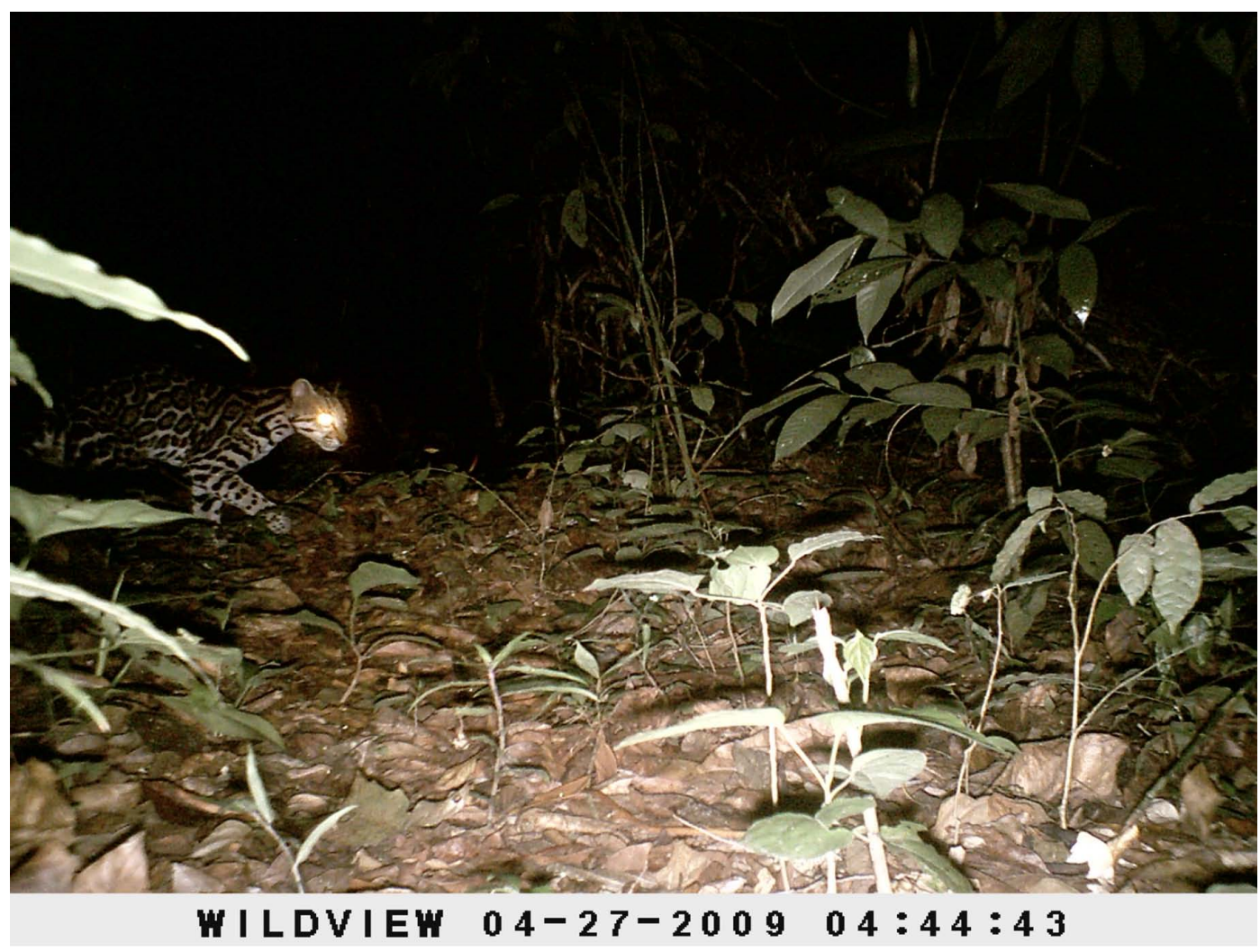

Este estudio, amplía la información sobre los carnívoros de Puebla y se ayuda a delimitar la distribución de estos dentro del estado. De encontrar otras especies tropicales dentro del estado, indicaría que se cuenta con una riqueza mayor a la que se creía. Lo anterior indicaría que, Puebla ocupa un lugar más importante a nivel nacional para la conservación.

Agradecemos a la National Geographic Society por el apoyo otorgado con la beca NGS YE Grant \#EC0390-08 y a la Universidad de las Américas, Puebla por las facilidades otorgadas para realizar este proyecto. En particular a las autoridades del municipio de Hueytamalco por todo el apoyo recibido para realizar este proyecto. 


\section{Referencias}

López-Wilchis, R., y J. López Jardines. 1998. Los mamíferos de México depositados en colecciones de Estados Unidos y Canadá. Universidad Autónoma Metropolitana, Unidad Iztapalapa, 323 pp.

Ramírez Pulido J., N. González-Ruiz, y H. H. Genoways. 2005. Carnivores from the mexican state of Puebla: distribution, taxonomy and conservation. Mastozoología Neotropical 12:37-52.

Villarreal Espino-Barros, O. A., R. Guevara, R. Reséndiz, J. S. Hernández, J. C. Castillo, y F. J. Tomé. 2005. Diversificación productiva en campo experimental Las Margaritas, Puebla, México. Archivos de Zootecnia 54:197-203.

Sometido: 14 octubre 2009

Revisado: 13 enero 2010

Aceptado: 16 febrero 2010

Editor asociado Sergio Ticul Álvarez-Castañeda 
